\title{
Noise pollution in hemodialysis centers
}

\author{
Claudio Ronco
}

Noise is an environmental pollutant and a potential health hazard. The incidence of several morbid conditions, such as depression, insomnia, and lack of concentration increases in urban areas where noise levels are high. Noise pollution in hospitals can be substantial-approaching the levels of chainsaws or jackhammers - making it nearly impossible for patients to rest and heightening their sensitivity to pain or discomfort. This holds true both for critically ill patients and for chronically ill patients admitted for repeated treatments such as hemodialysis.

Noise levels above 100-110 dB can be recorded in hemodialysis centers, and peak noise levels tend to occur at the beginning or end of dialysis shifts. Noise sources that affect patient comfort and staff performance in hemodialysis centers include slamming doors, rolling trolleys, alarms and pumps of dialysis machines, computers, water treatment systems (when they are contiguous to the treatment room), and air conditioners. Communication between staff members, conversations between patients, announcements through loudspeakers, and sounds from radios, TVs or other equipment not necessarily related to therapy (such as cell phones and beepers) are other sources of noise pollution in these centers.

We should strive to manage potential sources of noise pollution in hemodialysis centers by ensuring meticulous maintenance of dialysis machines and other equipment and by encouraging manufacturers to develop silent devices whenever possible. Such efforts should be especially made if machines are designed for home or nocturnal treatment. Patients, staff, and visitors in hemodialysis centers should be

\section{Noise pollution \\ in hospitals can be \\ substantial- approaching the levels of chainsaws or jackhammers...}

$C$ Ronco is Head of the Department of Nephrology, Dialysis and Transplantation at St Bortolo Hospital, Vicenza, Italy.

\section{Competing interests}

The author declared no competing interests.

www.nature.com/clinicalpractice doi:10.1038/ncpneph0829 encouraged to use lowered voices in conversation. The use of phones, beepers, and buzzers should be kept to a minimum, if not eliminated. Suitable windows and doors should be installed to minimize external noise. Structural components of hospital buildings, mobile equipment, door closures, and other sources of noise should be routinely inspected and subjected to rigorous maintenance. If patients wish to listen to music - which seems to reduce stress and improve relaxation and quality of sleep-it is probably better to encourage them to use headphones than to pipe music into public areas. Overall, the maximum continuous volume level in a hemodialysis center should be no more than $50 \mathrm{~dB}$.

Additionally, hemodialysis staff should aim to reduce noise when performing routine actions, such as delivering meals or cleaning, and when managing each patient's equipment. Checking and adjusting monitors to avoid unnecessary alarms, as well as choosing the right device to suit each individual patient, is important. Alarms tend to go off too frequently, especially at specific times of the shift, and are often too loud and not rapidly or effectively attended to.

The design of new hemodialysis centers should reflect the importance of noise reduction. A compromise should be reached between maintaining a soundproof environment and creating a space that is open enough so that several patients can be monitored at a glance. Incorporation of glass walls and other transparent barriers within such designs might well help in achieving this goal. The use of curtains and doors as sound barriers is controversial because of safety concerns, but these barriers might improve quality of life for the patient. 